\title{
Síndrome de Sturge-Weber: relato de caso clínico
}

\author{
Sturge-Weber Syndrome: report of a clinical case \\ Síndrome Sturge-Weber: reporte de un caso clínico
}

\section{Resumo}

A síndrome de Sturge-Weber é uma patologia congênita rara de desenvolvimento, não hereditária. É caracterizada por uma angiomatose cortical cerebral, com presença de calcificações cerebrais, epilepsia, nevus faciais e alterações oculares. O presente estudo teve como objetivo realizar um relato de caso de Síndrome de Sturge-Weber, em paciente atendido na clínica escola da Faculdade de Odontologia do Recife, apresentando manchas vinho do porto intrabucais, região da face, pavilhão auricular e região cervical, de localização unilateral, lado direito. Por ser síndrome rara, o presente relato de caso incorporado aos demais casos relatados na literatura, proporcionando maior conhecimento da classe odontológica sobre a doença, desde o auxílio ao diagnóstico aos cuidados necessários no tratamento odontológico dos pacientes portadores dessa síndrome.

Palavras-chave: Síndrome de Sturge-Weber; Síndromes neurocutâneas; Angiomatose.

\begin{abstract}
Sturge-Weber syndrome is a rare congenital developmental pathology that is not hereditary. It is characterized by cerebral cortex angiomatosis, with the presence of cerebral calcifications, epilepsy, facial nevus, and ocular alterations. The present study aimed to perform a case report of Sturge-Weber Syndrome, in a patient seen at the clinic school of the Recife School of Dentistry, presenting intraoral port wine staining, face region, pinna, and cervical region, unilateral location, right side. Because it is a rare syndrome, the present case report incorporated the other cases reported in the literature, providing more knowledge of the dental class about the disease, from the aid to diagnosis to the necessary care in the dental treatment of patients with this syndrome.
\end{abstract}

Keywords: Sturge-Weber syndrome; Neurocutaneous syndromes; Angiomatosis. 


\section{Resumen}

El síndrome de Sturge-Weber es una rara patología congénita del desarrollo que no es hereditaria. Se caracteriza por angiomatosis de la corteza cerebral, con presencia de calcificaciones cerebrales, epilepsia, nevus facial y alteraciones oculares. El presente estudio tuvo como objetivo realizar el reporte de un caso de Síndrome de Sturge-Weber, en un paciente atendido en la clínica escuela de la Facultad de Odontología de Recife, presentando manchas intraorales de vino de Oporto, región de la cara, pabellón auricular y en la región de la cara, el pabellón auricular y la región cervical, de localización unilateral, en el lado derecho. Por tratarse de un síndrome raro, el presente informe de caso incorporó los otros casos reportados en la literatura, proporcionando mayor conocimiento de la clase odontológica sobre la enfermedad, desde la ayuda al diagnóstico hasta los cuidados necesarios en el tratamiento odontológico de pacientes con este síndrome.

Palabras clave: Síndrome de Sturge-Weber; Síndromes neurocutáneos; Angiomatosis.

\section{Introdução}

A síndrome de Sturge-Weber (SSW), é uma doença neurocutânea congênita de rara ocorrência. É classificada como uma facomatose ou hamartomatose, sendo também denominada angiomatose encefalotrigeminal, comprometendo tecidos vasculares e nervosos (Figueiredo et al., 2011; Doumiri et al., 2020; Rachid et al., 2018). A SSW compreende um grupo de doenças neuroectodérmicas e mesodérmicas caracterizadas por lesões oculares congênitas e frequentemente hereditárias, associadas a lesões presentes em outros órgãos ou sistemas (Doumiri et al., 2020; Rodrigues, \& Prigenzi, 2013). Pacientes afetados por esta síndrome, demonstram malformações conhecidas como hamartomas, proliferações celulares anormais em tecidos habitualmente encontrados nas regiões ou nos órgãos afetados (Tomás-Vila et al.,2013; Comi, 2015; Rachid et al., 2018). A maioria das alterações é diagnosticada por mostrar alterações muito características na pele e no exame de fundo de olho (Figueiredo et al., 2011; Tomás-Vila, M., et al., 2013; Rodrigues, \& Prigenzi, 2013) . Apresenta três características clínicas conhecidas como tríade clássica: hemangiomas cutâneos, meníngeos e oculares (Cremé-Lambert et al., 2020; Higueros et al., 2017; Doumiri et al., 2020; Rachid et al., 2018). O angioma facial trata-se de uma malformação vascular, que não costuma regredir espontaneamente. Essa patologia acomete com maior frequência o primeiro e o segundo ramo do nervo trigêmeo $\left(\mathrm{V}_{1}\right.$ e $\left.\mathrm{V}_{2}\right)$, estendendo-se em alguns casos para a região cervical e tórax (Pila Pérez et al., 2010; Rodofile et al., 2011; Doumiri et al., 2020; Rachid et al., 2018). A SWS foi descrita em 1860 por Schirmer, um oftalmologista alemão, sendo apenas apresentada como patologia na íntegra em 1879 por Sturge. Após quarenta e três anos de estudo, em 1922, Weber completou a lista de sinais, descrevendo os resultados radiológicos de sua pesquisa (Maraña Pérez et al., 2017).

Sua etiologia é referida a uma mutação do mosaico somático em GNAQ (c.548G> A, p.R183Q) em lesões cerebrais e cutâneas (Higueros et al., 2017, Shirley et al., 2013) . Sem predileção por sexo, atinge igualmente homens e mulheres (Shirley et al., 2013). Sua incidência ocorre aproximadamente de $1 / 230000$ a 1/50000 nascidos vivos (Maraña Pérez et al., 2017). O diagnóstico é evidenciado a partir do exame clínico com a manifestação clínica de angioma facial e angiomatose leptomeníngea, confirmado por Tomografia Computadorizada Multislice, imagens de Ressonância Magnética e eletroencefalograma convencional (EEG) e vídeo-EEG onde são observadas as ondas de pico ou descargas de onda de poliponto nos lobos afetados pela angiomatose leptomeníngea (Higueros et al., 2017; Pila Pérez et al., 2010; Maraña Pérez et al., 2017; Rachid et al., 2018). As alterações vasculares podem afetar a cavidade bucal, produzindo hiperplasia vascular dos lábios, língua, mucosa oral, gengiva e periodonto (Pila Pérez et al., 2010; Rodofile et al., 2011; Shirley et al., 2013; Velásquez-Gallego et al., 2019).

As manchas cor vinho do porto (nevus flammeus) presentes na SSW podem ocorrer de forma isolada, localizados nas regiões na face, cabeça e pescoço, ou afetar ambos os lados da face e se estender ao tronco e outras extremidades (Santos \& Cavalheiro, 2010; Rodofile et. al., 2011; Doumiri et al., 2020). As manifestações neurológicas podem incluir cefaléia, convulsões, comprometimento neurológico e cognitivo, distúrbios da motilidade, retardo mental, hemiparesia, distúrbios do desenvolvimento e episódios de isquemia vascular com seus respectivos sintomas (Santos \& Cavalheiro, 2010; Sudarsanam \& Ardern-Holmes., 2014; Maraña Pérez et al., 2017). A angiomatose leptomeníngea sensibiliza a isquemia cortical crônica, que concebe áreas de calcificação e atrofia cerebral. Nos olhos, os angiomas provocam glaucoma, hemangioma episcleral ou 
conjuntival, hemangioma coroidal, heterocromia ou neovascularização da íris, hemianopsia homônima (Sudarsanam \& ArdernHolmes., 2014).

Não há tratamento específico para a Síndrome Sturge-Weber. O manejo clínico é realizado com uma equipe multidisciplinar necessária para monitoração da progressão da doença, durante toda a vida do paciente (Comi, 2015). As manchas vinho-do-porto, são tratadas com laserterapia, que devem ter início na infância, em estado inicial, enquanto a aparência é lisa e rosada, reagindo melhor à terapia e retardando a progressão e futuras complicações que afetam a visão, vias aéreas e a deglutição (Zallmann, M., et al., 2018; Neerupakam et al., 2017; Rachid et al., 2018). As convulsões são controladas para evitar comprometimento psicomotor no paciente, através de 1 ou 2 anticonvulsivantes associados a ácido acetilsalicílico (Rodofile, C., et al., 2011; Shirley et al., 2013; Velásquez-Gallego et al., 2019). Sendo os anticonvulsivantes mais prescritos: oxcarbazepina, carbamazepina, levetiracetam e fenobarbital (Zallmann et al., 2018; Neerupakam et al., 2017).

As manchas de vinho do Porto são caracterizadas por excessivos números de vasos sanguíneos dilatados na derme média e profunda. As lesões orais mostram semelhante dilatação vascular. Suas lesões gengivais proliferativas podem lembrar um granuloma piogênico (Neville, 2008). O tratamento odontológico dos pacientes acometidos da síndrome de angiomatose de Sturge-Weber pode ter repercussão na forma da condução do tratamento odontológico (Neerupakam et al., 2017), vez que o uso de convulsivantes podem levar a hiperplasia gengival. Essas más-formações que afetam a gengiva podem torná-la delicada, dificultando os procedimentos de profilaxia dentária. Procedimentos cirúrgicos em áreas afetadas podem causar intensa hemorragia (Neville, 2008).

Este estudo tem como objetivo apresentar um relato de caso clínico de paciente portador de Síndrome de Sturge-Weber, referente ao acompanhamento clínico hospitalar e assistência odontológica pelo cirurgião-dentista e equipe multidisciplinar, sobre o conhecimento da patologia, suas manifestações clínicas e condutas de tratamento.

\section{Metodologia}

Este artigo trata-se de um relato de caso, conduzido de forma descritiva e qualitativa. Segundo Pereira et al., (2018), configura-se uma pesquisa por via direta que busca recolher dados relativos ao estudo através do acesso aos registros e aos exames fornecidos, sendo o pesquisador a ferramenta primordial. O presente estudo trata-se de um relato clínico de Síndrome de Sturge-Weber. Todas as informações em questão foram colhidas através do prontuário do paciente, além de imagens fotográficas com o intuito de descrever o caso. No que se relacionam aos aspectos éticos, esclarecimentos sobre riscos, benefícios e prognósticos foram fornecidos ao paciente por meio do Termo de Consentimento Livre e Esclarecido (TCLE) e a autorização do procedimento ocorreu mediante assinatura de tal documento.

\section{Relato de Caso Clínico}

Paciente, sexo masculino, 29 anos, apresentando Estado Geral Regular, consciente, orientado, natural de Recife — Pernambuco - Brasil, compareceu ao serviço de atendimento odontológico da Clínica Escola da Faculdade de Odontologia do Recife, Fundação Odontológica Presidente Castelo Branco — FOPCB, em Recife — Pernambuco-Brasil, sem acompanhante, tendo como queixa principal dor de dente, aproximadamente 06 meses e queixando-se também da sua estética bucal. Referiu sensibilidade nos elementos dentários inferiores, principalmente na região de molares, ao consumir alimentos frios. Queixou-se ainda de sangramento gengival na escovação e de forma espontânea. Informou ainda que tinha dificuldade de mastigar. Na história da doença atual, relatou que as manchas vermelhas na sua face existem desde o nascimento. Teve acompanhamento médico desde então, segue acompanhado pela clínica médica (neurologia, dermatologia e cirurgia vascular). Referiu ainda que faz uso contínuo de anticoagulante e carbamazepina como tratamento da patologia, prescrito pela clínica médica.

Ao exame físico extrabucal, foram observadas manchas na cor violácea (vinho-do-Porto) nas regiões de face, couro 
cabeludo, orelha e pescoço do lado direito em regiões inervadas pelo nervo trigêmeo. (Figura 1 A, B e C).

Figura 1 A, B e C. Perfil e face frontal com mancha cor de vinho distribuída nos territórios V1 e V2.
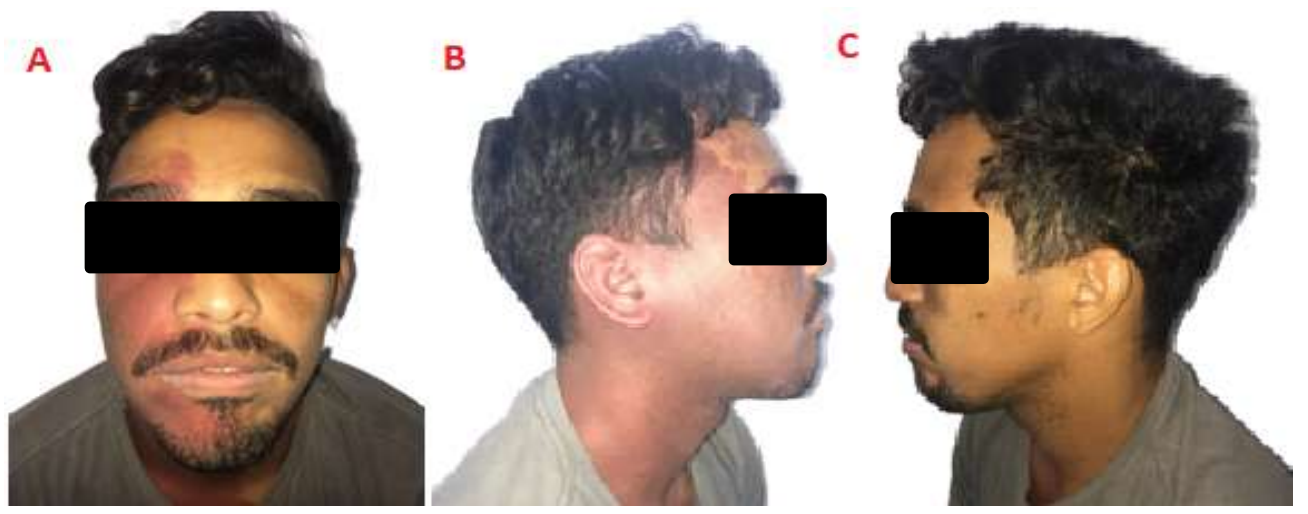

Fonte: Autores (2020).

Ao exame físico intrabucal, apresentava gengiva hiperplásica de coloração vinhosa unilateral em arcos superior e inferior do lado direito, com presença de sangramento e sensibilidade, como também unilateralmente no assoalho da boca e língua, região de palato evoluindo para orofaringe (Figura 2 A e B).

Figura 2A e B. Aspecto intrabucal.

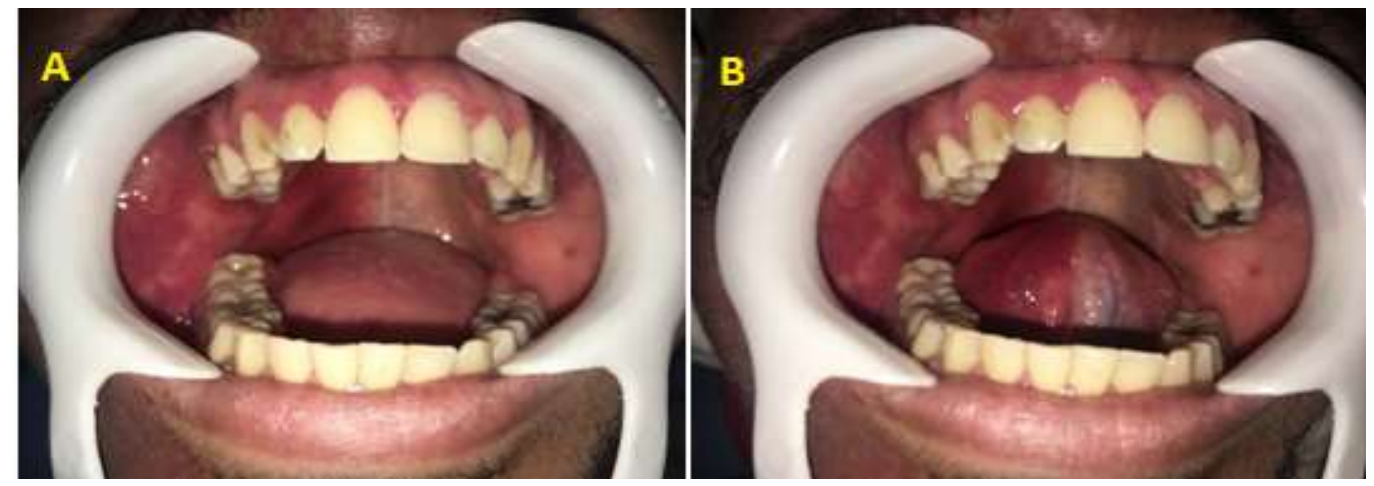

Fonte: Autores (2020).

No ato da consulta, o paciente apresentou um laudo médico de sua história clínica com todo o histórico fornecido pela clínica médica. Informando que o paciente já nasceu portador de Síndrome de Sturge-Weber (SSW), tendo início com crises epilépticas aos 04 meses de vida, de tipo parcial (com presença de desvios dos olhos e repetindo palavras), apresentando bom desenvolvimento motor normal (andou com 1 ano), apresentando hemianopsia homônima esquerda, quando pisca à ameaça. Em Tomografia Computadorizada Multislice realizada em 01 ano de vida, apresentou angioma leptomeníngeo parietal posterior direito, com controle ao tratamento com Carbamazepina $25 \mathrm{mg} / \mathrm{kg} / \mathrm{dia}$ e $10 \mathrm{mg}$ de clobazam $/$ dia. Aos 05 anos foi notado distúrbios de aprendizagem, como não sabia identificar e nomear as cores. Em avaliação oftalmológica em abril/1998, não apresentou evidências de alterações. Após apresentar crise convulsiva associada a trauma crânio encefálico, apresentou sonolência, episódios de vômito, e presença de crises mioclônicas, frequentes, cedendo apenas com sedação através de emprego de fenitoína e midazolam, prescrito à época pelo médico emergentista que prestou seu atendimento. Em eletroencefalograma em 
novembro/1998, apresentou descargas paroxísticas difusas de ondas agudas de moderada amplitude, potencialmente epileptogênicas. O paciente vem sendo acompanhado pelo psiquiatra desde 1999, por problemas relacionados à doença. Devido ao uso constante de carbamazepina, para controle das crises epiléticas, apresentou um déficit escolar importante, pois embora frequentasse a quinta série escolar, não sabia ler e nem fazer cálculos simples, diagnosticado também com Glaucoma.

Em outubro de 2012, em exame de angiografia cerebral não foram observados aneurismas e/ou malformações vasculares, onde houve então a confirmação do diagnóstico de Síndrome de Sturge-Weber sem malformação vascular cerebral. Encaminhado ao dermatologista para tratamento com fotocoagulação à ("laser") para as manchas de vinho do porto. Em exame, feito à época, de ressonância magnética do crânio, foram observados sinais de redução volumétricas dos giros corticais da região parietal e parieto-occipital à direita com áreas de baixo sinal subcorticais sugestivas de calcificação córtico-subcorticais, considerado devido à Síndrome de Sturge-Weber. Em Tomografias computadorizadas de crânio sem contraste, foi evidenciado a presença de alteração leptomeníngea (Figura 3 A, B, C e D; Figura 4 A e B).

Figura 3 A, B, C e D. Presença de angioma leptomeníngeo parietal posterior direito.

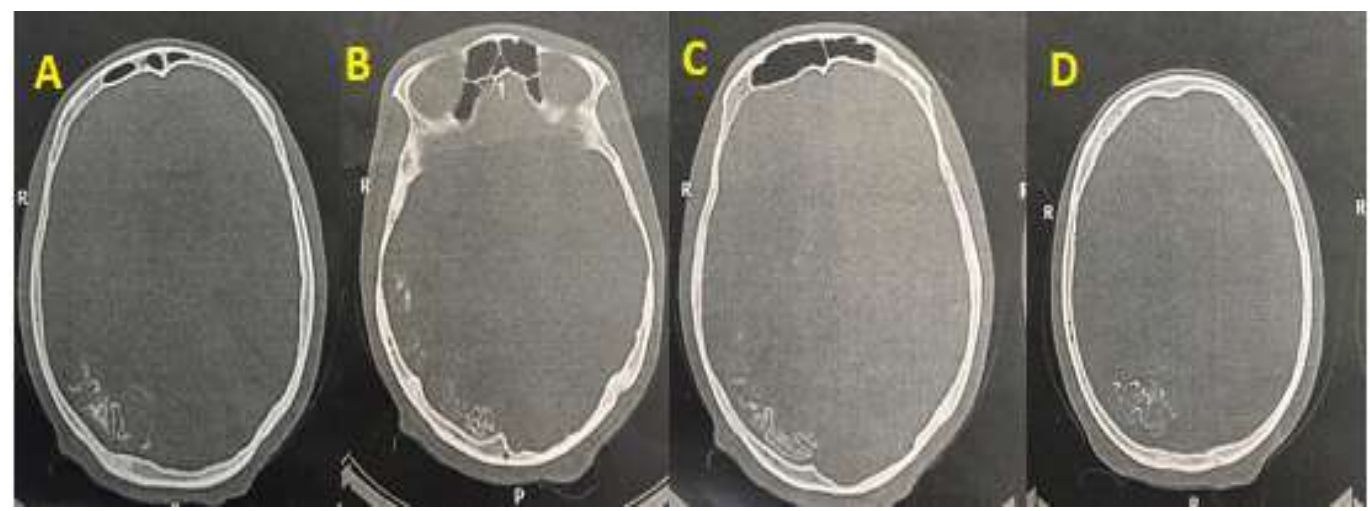

Fonte: Autores (2020).

Figura 4 A e B. Cortes axiais de tomografias computadorizadas do crânio.

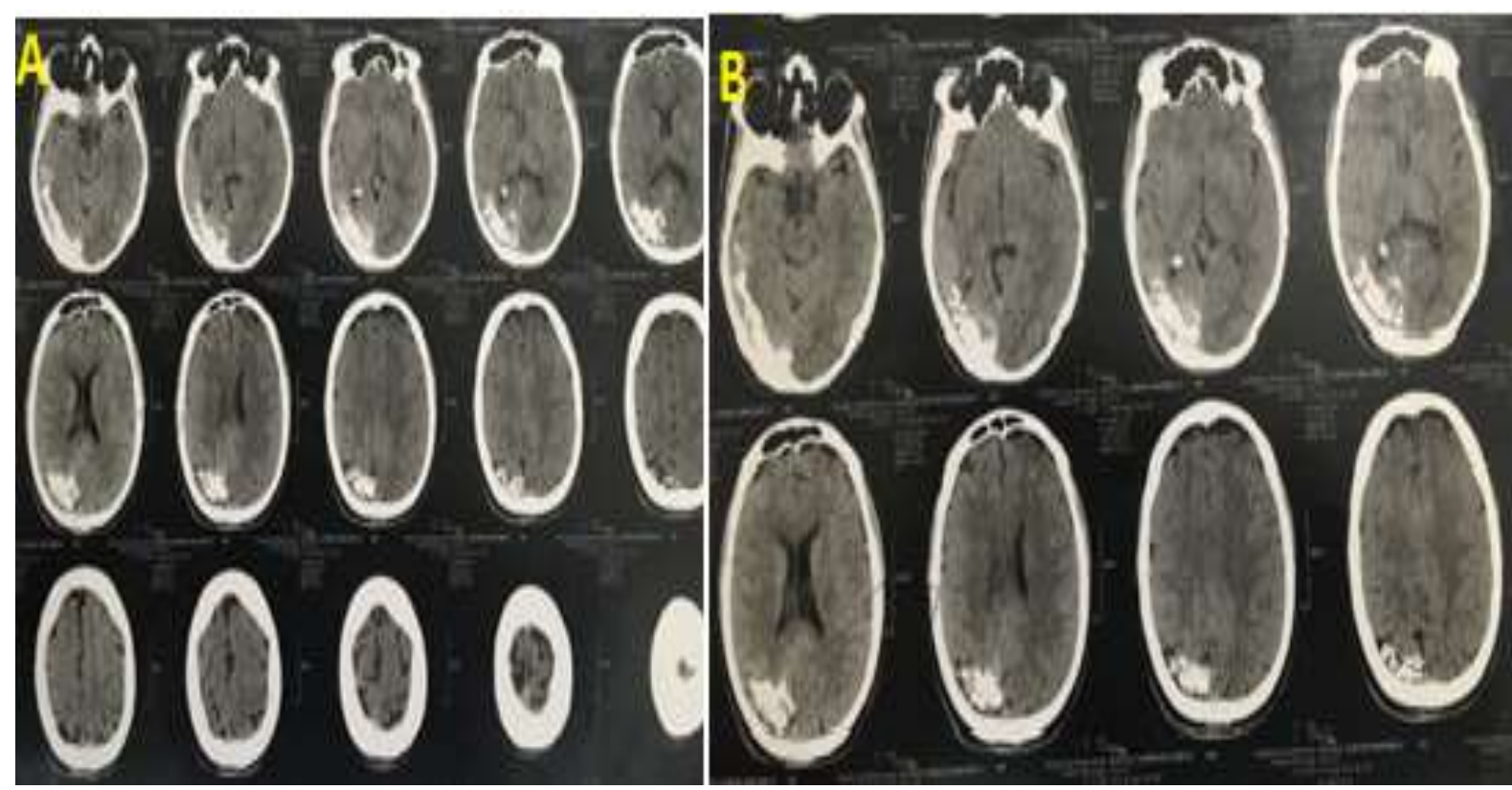

Fonte: Autores (2020). 
O paciente foi submetido a tratamento odontológico clínico não invasivo, devido ao risco de hemorragia, apenas realizado a profilaxia dentária mais fluorterapia e restauração do elemento dentário 46 com resina composta que apresentava restauração de amálgama com infiltração, o que acarretava sensibilidade dentária do paciente. A SSW pode influenciar na conduta de tratamento do cirurgião-dentista, pois o paciente faz uso contínuo de anticonvulsivante, além do risco de hemorragia devido às lesões vasculares, procedimentos invasivos como raspagem sub e supragengival, gengivoplastia, gengivectomias, exodontias, biópsias e remoção de cistos e tumores odontogênicos, considerados de riscos a esses pacientes. O paciente a princípio é bom higienista, não possui presença de tártaro ou placa bacteriana, pouca incidência de cáries, apenas com sangramento anormal ao considerado patológico da gengivite devido à presença das lesões vasculares, acompanhado pela Odontologia e orientado ao uso de enxaguatórios bucais com clorexidina e instruções de higiene oral.

\section{Discussão}

A síndrome de Sturge-Weber (SSW) é uma displasia neuroectodérmica, que compromete a região facial inervada pelo nervo trigêmeo, desde a pálpebra superior e a região supraorbital, aos ramos maxilar e mandibular do referido nervo (Figueiredo et al., 2011; Tomás-Vila et al., 2013; Cremé-Lambert et al., 2020; Doumiri et al., 2020).

Em recém-nascidos, manifesta-se em cerca de $0,3 \%$ dos casos. A angiomatose facial é localizada na região frontal e pálpebras, no primeiro e segundo ramo do nervo trigémeo. A lesão se caracteriza como plana e rosada, onde à medida que a criança cresce, a cor se modifica para o vermelho ao vinho, em alguns casos as lesões vasculares podem causar nodularidade superficial e bolhas (Maraña Pérez et al., 2017; Avalos-Lara et al., 2020).

Segundo Rodofile et. al., 2011, cerca de 37\% dos pacientes são diagnosticados com lesões bilaterais podendo estar associadas a acometimento intracraniano unilateral ou bilateral, enquanto $36 \%$ são diagnosticados com malformações vasculares em região cervical ou torácica. Neste relato de caso, o paciente apresentava lesões unilaterais, apenas. Casos de localização atípica, como couro cabeludo ou mucosa oral, fora da região do trigêmeo, estão associadas a alteração leptomeníngea, o que foi observado no caso clínico apresentado: o paciente apresentava nevus flammeus unilateralmente do lado direito da face, intraoral, em pescoço e na região de orelha, couro cabeludo e neurocrânio, associado a presença de angioma leptomeníngeo parietal posterior direito.

Segundo Neerupakam et al., 2017, associadas às lesões bucais são encontradas principalmente alterações vasculares dos lábios e da mucosa oral, crescimento ósseo ou de tecidos moles e espessamento gengival, essas manifestações orais são geralmente unilateral e terminam abruptamente na linha média. A macroglossia e a hipertrofia do osso maxilar, encontrada em alguns pacientes, pode causar má oclusão e assimetria facial (Gomes et al., 2004). A hiperplasia gengival nestes pacientes está relacionada à terapia anticonvulsivante, o que foi visualizado neste relato de caso, onde o nevus flammeus facial no território trigeminal estavam também associadas alterações neurológicas, prescrito o anticonvulsivante de uso contínuo pela clínica médica.

Segundo Pérez et al., 2010, e Neerupakam et al., 2017, entre os diagnósticos diferenciais podemos citar: facomatose pigmentar vascular; Parkes-Weber, Rubinstein-Taybi, síndrome PHACES, Klippel-Trenaunay-Weber; Rendu-Osler-Weber; Von Hippel-Lindau; Wyburn-Mason; Shapiro-Shulman; Divry-Van Boagaert; Bannayan-Zonna and Cobb Syndrome. Segundo Higueros et al., 2017, a tomografia computadorizada (TAC) de crânio e face ou as imagens de ressonância magnética, podem sugerir o diagnóstico precoce do início das manifestações clínicas neurológicas, tendo sido esses dados que levaram inicialmente os médicos acompanhantes a diagnosticarem desde cedo a síndrome.

Segundo Tomás-Vila et al., 2013, na síndrome de Sturge-Weber cerca de $60 \%$ das crianças têm problemas de desenvolvimento, aprendizagem e comportamento. Nestes casos, pode haver delírios e alucinações, que estão relacionadas à extensão da lesão cerebral e à gravidade e idade de início das convulsões, mais comuns na infância. Neste relato foram coletados 
dados referentes a distúrbios de aprendizagem e de comportamento aos 05 anos, onde apresentou dificuldade em identificar e nomear as cores.

$\mathrm{Na}$ SSW, o tratamento em geral inclui anticonvulsivos para controle de epilepsia, tratamento sintomático e profilático para cefaléia e glaucoma para reduzir a pressão intraocular (Avalos-Lara et al., 2020). O paciente faz uso contínuo de anticonvulsivante e ácido acetilsalicílico e tem glaucoma controlado pelo oftalmologista.

Segundo Higueros et al., 2017, o tratamento com "laser" corante pulsado (PLD) é o tratamento de escolha para as manchas de vinho do porto na face. Sendo necessárias entre 7 e 15 sessões para limpar as lesões e raramente as lesões desaparecem completamente. A resposta clínica depende da cor inicial da lesão e de sua localização. A região frontal responde melhor do que na região malar ou labial. A laserterapia é eficaz no tratamento deste tipo de lesões faciais, contudo, devido à elevada taxa de recorrência causada pela reperfusão dos vasos, Maraña Pérez et al., 2017 sugerem novas técnicas de estudos, combinando o uso de rapamicina tópica com "laser".

A trombose recorrente trata-se de uma complicação resultante a estase venosa devido a mecanismo subjacente da patogênese da SSW. A utilização de agentes antiplaquetários para reduzir a trombose é uma terapia recomendada, que na odontologia pode trazer certas complicações aos pacientes, durante o procedimento invasivo. O uso de aspirina associado a um curso estável da SSW reduz as crises convulsivas, epilépticas e episódios de AVC. Porém devemos salientar que o uso de aspirina em dose baixa na SSW é controverso, pois a relação risco-benefício permanece por estabelecer, e preocupante quanto aos potenciais riscos de síndrome de Reye. A aspirina reduz a frequência das convulsões em portadores de SSW, por meio do seu mecanismo de ação antiplaquetário, evitando a formação de trombos, trazendo melhorias na circulação dos pacientes (Bay, MJ., et al., 2011).

Na clínica odontológica, muito embora de rara ocorrência, é possível nos deparamos com pacientes portadores de lesões hemangiomatosas. Nesse caso, é importante atentar o Cirurgião-Dentista que os pacientes acometidos da SSW podem apresentar sinais fundamentais da patologia, muitas vezes acompanhados de manifestações convulsivas e epilépticas. A maioria dos pacientes, por usarem Fenobarbital podem apresentar hiperplasia gengival (Gomes et al., 2004). É necessário que o profissional tenha conhecimento específico para realização de assistência em saúde e assistência odontológica a pacientes portadores de Síndrome de Sturge-Weber, promovendo assim a saúde e o bem-estar do indivíduo, prevenindo complicações. A angiomatose intra-oral causa alterações periodontais que aumentam o risco de hemorragia durante procedimentos dentários e intubação orotraqueal (Maraña Pérez et al., 2017).

Isso só enaltece a necessidade do conhecimento da patologia pelo Cirurgião-Dentista, devendo o mesmo ter cuidado com procedimentos invasivos nas áreas afetadas por conta de hemorragias, controle periodontal contínuo para minimizar os efeitos periodontais dos anticonvulsivantes e ter o cuidado especial de, ao realizar o atendimento odontológico, entrar em contato com o neurologista do paciente de modo a verificar se há necessidade de aumento da dose do anticonvulsivante quando realizar procedimentos extensos. O Cirurgião-Dentista deve evitar incidir diretamente o foco luminoso sobre os olhos do paciente, evitando desencadear crises epilépticas.

\section{Considerações Finais}

No acompanhamento e assistência odontológica a pacientes portadores de Síndrome de Sturge-Weber, é de vital importância que o cirurgião-dentista tenha conhecimento do que é a doença, suas manifestações clínicas e como manejar o paciente acometido por essa categoria de enfermidade, já que ao executar alguns procedimentos em pacientes portadores da SSW é necessária uma avaliação cautelosa no planejamento de tratamento a ser estipulado, observando-se os riscos de complicações sistêmicas e de ordem local (hemorragias) durante e após procedimentos invasivos. 
Research, Society and Development, v. 10, n. 16, e120101623327, 2021

(CC BY 4.0) | ISSN 2525-3409 | DOI: http://dx.doi.org/10.33448/rsd-v10i16.23327

\section{Agradecimentos}

Ao professor Sérgio Martorelli pela dedicação e amor ao ensino odontológico.

\section{Referências}

Avalos-Lara, S. J., Antonio-Aguirre, B., Perez-Ortiz, A. C., Mendoza Velásquez, C., Camacho-Ordoñez, A., \& Palacio Pastrana, C. (2020). Spherophakia and Ectopia Lentis in a Sturge-Weber Patient: A Case Report. Case reports in ophthalmology, 11(2), 356-363.

Bay, M. J., Kossoff, E. H., Lehmann, C. U., Zabel, T. A. \& Comi, A. M. (2011). Pesquisa do uso de aspirina na síndrome de Sturge-Weber. Journal of Child Neurology, 26(6), 692-702.

Cremé-Lambert, L., Díaz-Estévez, H., \& Lamas-Ávila, M. (2020). Síndrome de Sturge-Weber. Revisão da literatura e apresentação de um caso. Scientific Information Magazine, 99 (1), 89-101.

Comi A. M. (2015). Sturge-Weber syndrome. Handbook of clinical neurology, 132, 157-168

Doumiri, M., Labied, M., Salam, S., Laoudiyi, D., Chbani, K., \& Ouzidane, L. (2020). Le syndrome Sturge-Weber: à propos d'un cas [Sturge-Weber syndrome: about a case]. The Pan African medical journal, 36, 273.

Figueiredo, L. R. C., Silva Filho, F. J. d., \& Rehder, J. R. C. L. (2011). Síndrome de Sturge-Weber e suas repercussões oculares: revisão da literatura. Revista Brasileira de Oftalmologia, 70(3), 194-199.

Gomes, A. C. A., Dias, E. D. O. E. S., \& Albert, D. G. D. M. (2004). Síndrome de Sturge-Weber: Relato De Caso Clínico. Rev. Cir. Traumatol. Buco-MaxiloFac, 4(1), 47-52.

Higueros, E., Roe, E., Granell, E., \& Baselga, E. (2017). Síndrome de Sturge-Weber: uma revisão. Síndrome de Sturge-Weber: revisión. Actas dermosifiliograficas, 108 (5), 407-417.

Lance, E. I., Sreenivasan, A. K., Zabel, T. A., Kossoff, E. H. \& Comi, A. M. (2012). Uso de aspirina na síndrome de Sturge-Weber. Journal of Child Neurology, 28(2), 213-8.

Maraña Pérez, A. I., Ruiz-Falcó Rojas, M. L., Puertas Martín, V., Domínguez Carral, J., Carreras Sáez, I., Duat Rodríguez, A., \& Sánchez González, V. (2017). Analysis of Sturge-Weber syndrome: A retrospective study of multiple associated variables. Análisis del síndrome de Sturge-Weber: estudio retrospectivo de múltiples variables asociadas. Neurologia (Barcelona, Spain), 32(6), 363-370.

Neerupakam, M., Reddy, P. S., Babu, B. A., \& Krishna, G. V. (2017). Síndrome de Sturge Weber: um estudo de caso. Journal of Clinical and Diagnostic Research, 11(5).

Neville, B., Damm, D., Allen, C., \& Bouquot, J. (2008). Tumores dos tecidos moles. In: Patologia Oral \& Maxilofacial. (2a ed.). Guanabara.

Pereira, A. S., Shitsuka, D. M., Parreira, F. J., \& Shitsuka, R. (2018). Metodologia da pesquisa científica. [free e-book]. Santa Maria/RS. Ed. UAB/NTE/UFSM

Pila Pérez, R., Conde Rivera, M. E., Pila Pélaez, R., Holguín Prieto, V., \& Torres Vargas, E. (2010). Síndrome de Sturge-Weber: apresentação de um caso. Camagüey Medical Archive Magazine, 14 (5)

Rachidi, S. A., Mimi, A. L., Akammar, A., Alaoui, Y. L., Boubbou, M., Maaroufi, M., \& Alami, B. (2018). Syndrome de Sturge Weber Krabbe: entité exceptionnelle (à propos d'un cas) [Sturge-Weber-Krabbe Syndrome: an uncommon disorder (about a case)]. The Pan African medical journal, $31,211$.

Rodofile, C., Grees, S. A., Valle, L. E., \& Martino, G. (2011). Síndrome de Sturge-Weber: Apresentação de um caso com manifestações dermatológicas mínimas. Arquivos Pediátricos Argentinos, 109 (2), 42-45.

Rodrigues, B. R., \& Prigenzi, M. L. H. (2013). Síndrome de Sturge-Weber. Revista Da Faculdade De Ciências Médicas De Sorocaba, 15(3), 76-78.

Santos, S. V. P., \& Cavalheiro, L. G. (2010). Síndrome de Sturge-Weber: relato de caso dos achados da avaliação fonoaudiológica. Revista CEFAC, 12(1).

Shirley, M. D., Tang, H., Gallione, C. J., Baugher, J. D., Frelin, L. P., Cohen, B., North, P. E., Marchuk, D. A., Comi, A. M., \& Pevsner, J. (2013). SturgeWeber Syndrome and Port-Wine Stains Caused by Somatic Mutation in GNAQ. The new england jo u r na $l$ of $m$ e dic $i$ n e, 368(21).

Sudarsanam, A., \& Ardern-Holmes, S.L. (2014). Síndrome de Sturge-Weber: do passado ao presente. European Journal of Pediatric Neurology, 18 (3), 257266.

Tomás-Vila, M., Menor-Serrano, F., Arcos-Machancoses, J. V., García-Camuñas, Y., Pitarch-Castellanos, I., \& Barbero, P. (2013). Deficits focales transitorios en ninos afectados por el sindrome de Sturge-Weber [Transient focal deficits in children with Sturge-Weber syndrome]. Revista de neurologia, 56(4), 253-255.

Velásquez-Gallego, C., Ceballos-Ruiz, J. F., Ruiz-Jaramillo, N., \& Villamizar-Londoño, C. (2019). Síndrome de Sturge-Weber: Um Relato de Caso e Revisão da Literatura. Ecuadorian Journal of Neurology, 28 (2), 105-114.

Zallmann, M., Mackay, M. T., Leventer, R. J., Ditchfield, M., Bekhor, P. S. \& Su, J. C. (2018). Revisão retrospectiva da triagem para síndrome de Sturge-Weber com ressonância magnética do cérebro e eletroencefalografia em bebês com manchas em vinho do porto de alto risco. Dermatologia pediátrica. 35(5), 575-81. 